\title{
Symmetry Reduction for Stochastic Hybrid Systems
}

\author{
Manuela L. Bujorianu and Joost-Pieter Katoen
}

\begin{abstract}
This paper is focused on adapting symmetry reduction, a technique that is highly successful in traditional model checking, to stochastic hybrid systems. We first show that performability analysis of stochastic hybrid systems can be reduced to a stochastic reachability analysis (SRA). Then, we generalize the notion of symmetry reduction as recently proposed for probabilistic model checking, to continuous probabilistic systems. We provide a rigorous mathematical foundation for the reduction technique in the continuous case and also investigate its observability perspective. For stochastic hybrid systems, characterizations for this reduction technique are provided, in terms of their infinitesimal generator.
\end{abstract}

Keywords: Markov models, symmetries, transformation group, abstractions, reachability, probabilistic model checking.

\section{INTRODUCTION}

Symmetry reduction is a well-investigated technique for combatting the impact of state-explosion in temporal logic model checking (see [8], [10] and the references therein). This method exploits the occurrence of replication in a model. It has been applied mainly to models of concurrent systems of processes, such as communication and memory consistency protocols. Symmetry reduction gives the possibility to verify a model over a reduced quotient model, which is not only much smaller, but also bisimulation-equivalent to the original.

In the continuous setting, symmetry reduction techniques appear in different contexts. The collection of the planar motions that keep a geometric figure invariant form a group, called the symmetry group of the figure (rectangle, triangle, circle). It gives a measure for the symmetry degree of the figure, and it might help to reconstitute it from its parts. For an algebraic equation, a symmetry group is composed by the base space transformations that permute solutions. In the case of ordinary differential equations (ODE), all the special techniques for solving certain classes of ODE have their origin in a general method related to the existence of a continuous invariance group for these ODE. A unifying framework that carries out the hybrid geometric reduction of deterministic hybrid systems, generalizing classical reduction to a hybrid setting has been developed in [1], [7]. In the stochastic continuous case, symmetry features have been also employed in different frameworks. The symmetries of the Laplacian on the Euclidean space are of great help for studying properties of the Brownian motion. The diffusion processes having the maximal symmetry properties are characterised in [9].

M.L.. Bujorianu is with CICADA, University of Manchester, The UK. Manuela.Bujorianu@manchester.ac.uk

J.-P. Katoen is with RWTH Aachen University, Software Modelling and Verification Group, D-52056 Aachen, Germany katoen@informatik.twth-aachen.de
In this paper, we generalize the symmetry reduction techniques as recently proposed for probabilistic model checking, to continuous probabilistic systems (briefly presented in Section IV). The main purpose of our investigations is to apply these techniques to stochastic hybrid systems [4]. For continuous time/space Markov processes, when we generalise the symmetry reduction technique from [8], we obtain nothing else, but the space reduction using invariance transformation groups beautifully exposed by Dynkin, E.B in [6], Ch. 10 (see content of Section V). The main difficulty in applying such a technique to stochastic hybrid systems is to find out the appropriate invariance transformations that act uniformly on the domains of different discrete modes (with corresponding diffusion processes and guards), and is compatible with the jumping part. This jumping part is given by the discrete transitions between modes and is governed by some rates and reset maps. To overcome this problem, we propose a novel approach for the symmetry reduction of the state space of a Markov process considering transformation groups that preserve 'observations' over the trajectories. We provide a rigorous mathematical foundation for this reduction technique and also prove that the reduced quotient model is bisimulation-equivalent to the original model (Section VI). Section VII is dedicated to applying these techniques to stochastic hybrid systems.

\section{Probabilistic Models}

A probabilistic model is a transition system with the state space $X$, whose behaviour is specified not by a transition relation on $X$, but a transition function. The most known probabilistic models are: discrete-time Markov chains (DTMC), continuous-time Markov chains (CTMC).

\section{A. Discrete/Continuous-time Markov chains}

DTMCs are defined by a function $P: X \times X \rightarrow[0,1]$ satisfying $\sum_{x^{\prime} \in X} P\left(x, x^{\prime}\right)=1$ for each $x \in X$. This function is known as the transition probability matrix, gives the probability $P\left(x, x^{\prime}\right)$ of making a transition from each state $x$ to any other state $x^{\prime}$. CTMCs are defined by a transition rate matrix $R: X \times X \rightarrow \mathbb{R}_{+}$giving the rate $R\left(x, x^{\prime}\right)$ at which transitions between state pairs $\left(x, x^{\prime}\right)$ occur. This rate is interpreted as the parameter of a negative exponential distribution, resulting in a dense model of time. A CTMC is defined on a denumerable state space $X$ and with the stochastic transition matrix $P(t)=\left(p_{x y}(t)\right)$, where $x$ and $y$ range over $X$. Let us denote by $Q=\left(q_{x y}\right)$ the righthand derivative at $t=0$ of $P(t)$, i.e. the generator matrix of the chain. The entries of the infinitesimal generator matrix $Q$ are the rates at which the process jumps from state to state. 


\section{B. Continuous time/space Markov processes}

The stochastic processes we consider here are randomized systems with a continuous state space, where the "noise" can be measured using transition probability measures. Markov processes form a subclass of stochastic systems for which, at any stage, future evolutions are conditioned only by the present state.

State Space The state space is denoted by $X$. Suppose that $X$ is a Polish or analytic space ${ }^{1}$. We consider $X$ equipped with its Borel $\sigma$-algebra $\mathcal{B}$ (i.e. the $\sigma$-algebra generated by all open sets). The set of all bounded measurable numerical functions on $X$ is denoted by $\mathbf{B}(X)$. This set can be thought of as an additive monoid $S=(\mathbf{B}(X),+, 0)$ or a Banach space with the norm given by the supremum.

Sample Probability Space A probability space $(\Omega, \mathcal{F}, P)$ is fixed and all $X$-valued random variables are defined on this probability space. The trajectories in the state space are modelled by a family of random variables $\left(x_{t}\right)$ where $t$ denotes the time. The reasoning about state change is carried out by a family of probabilities $P_{x}$ one for each state $x \in X$. With any state $x \in X$ we can associate a natural probability space $\left(\Omega, \mathcal{F}, P_{x}\right)$ where $P_{x}$ is a probability measure such that $P_{x}\left(x_{t} \in A\right)$ is $\mathcal{B}$-measurable in $x \in X$, for each $t \in$ $[0, \infty)$ and $A \in \mathcal{B}$, and its initial probability distribution is $P_{x}\left(x_{0}=x\right)=1$. An extra point $\partial$ (the cemetery or deadlock point) is added to $X$ as an isolated point, $X_{\partial}=X \cup\{\partial\}$. Let $\mathcal{B}\left(X_{\partial}\right)$ be the Borel $\sigma$-algebra of $X_{\partial}$. The 'termination time' $\zeta(\omega)$ is the random time when the process $M$ escapes to and is trapped at $\partial$.

Strong Markov Property Formally, let $M=$ $\overline{\left(\Omega, \mathcal{F}, \mathcal{F}_{t}, x_{t}, P, P_{x}\right)}$ be a strong Markov process with the state space $X$, and with underlying probability space $(\Omega, \mathcal{F}, P) . \mathcal{F}_{t}$ describes the history of the process up to the time $t\left(\mathcal{F}_{t}\right.$ is the $\sigma$-algebra generated by the random variables $x_{s}, s \leq t$ ). Strong Markov property means that the Markov property is still true w.r.t. the stopping times of the process $M$.

Transition Function A transition function $p_{t}(x, \Gamma)$ is a transition probability function for a time homogeneous Markov process if $P\left\{x_{t+s} \in \Gamma \mid \mathcal{F}_{t}\right\}=p_{s}\left(x_{t}, \Gamma\right)$, for all $s, t \geq 0$ and $\Gamma \in \mathcal{B}(X)$.

Semigroup of operators The base of Markov process analysis is given by the concept of operator semigroup: $P_{t} f(x)=$ $E_{x} f\left(x_{t}\right), t \geq 0$. The right-hand derivative of $P_{t}$ for $t=0$ is called the infinitesimal operator (or generator) of the process. The infinitesimal generator of $\mathcal{P}=\left(P_{t}\right)$ is the possibly unbounded linear operator $\mathcal{A}$ defined by: $\mathcal{L} f=\lim _{t \searrow 0} \frac{P_{t} f-f}{t}$. The domain $D(\mathcal{L})$ is the subspace of $\mathbf{B}(X)$ for which this limit exists. Under very broad assumptions, the infinitesimal operator uniquely determines the transition function of the process.

Shift Operator For each $t \geq 0$ there exists a map $\theta_{t}: \Omega \rightarrow \Omega$

\footnotetext{
${ }^{1} \mathrm{~A}$ Polish space is a topological space, which is a homeomorphic image of complete separable metric space. The continuous image of a Polish space is called an analytic space.
}

called shift operator or simply shift such that

$$
x_{s} \circ \theta_{t}=x_{s+t}, \forall s \geq 0 .
$$

\section{Stochastic Hybrid Systems}

We adopt the General Stochastic Hybrid System model presented in [4]. This subsection describes the model and establishes the notation.

Let $Q$ be a set of discrete states. For each $q \in Q$, we consider the Euclidean space $\mathbb{R}^{d(q)}$ with dimension $d(q)$ and we define an invariant as an open subset $X^{q}$ of $\mathbb{R}^{d(q)}$. The hybrid state space is the set $X(Q, d, \mathcal{X})=\bigcup_{i \in Q}\{i\} \times X^{i}$ and $x=\left(i, z^{i}\right) \in X(Q, d, \mathcal{X})$ is the hybrid state. The closure of the hybrid state space will be $\bar{X}=X \cup \partial X$, where $\partial X=$ $\bigcup_{i \in Q}\{i\} \times \partial X^{i}$.

A (General) Stochastic Hybrid System (SHS) is a collection $H=((Q, d, \mathcal{X}),(b, \sigma)$, Init,$(\lambda, R))$, where: $(Q, d, \mathcal{X})$ describes the hybrid state space; $(b, \sigma)$ provides the coefficients of the diffusion part; Init is the initial probability measure defined on $(X, \mathcal{B}(X)) ;(\lambda, R)$ gives the jumping mechanism: $\lambda: \bar{X}(Q, d, \mathcal{X}) \rightarrow \mathbb{R}^{+}$is a transition rate function; $R: \bar{X} \times \mathcal{B}(\bar{X}) \rightarrow[0,1]$ is a stochastic kernel that provides the post-jump location [4]. The realization of an SHS is built as a Markov string obtained by the concatenation of the paths of some diffusion processes $\left(z_{t}^{i}\right)$, $i \in Q$ together with a jumping mechanism given by a family of stopping times $\left(S^{i}\right)$ [4]. The realization of any SHS, $H$, under standard assumptions (about the diffusion coefficients, non-Zeno executions, transition measure) is a strong Markov process $M=\left(\Omega, \mathcal{F}, \mathcal{F}_{t}, x_{t}, P_{x}\right)$. The sample paths of $M$ are right continuous with left limit, i.e. cadlags.

\section{Stochastic ReACHABILITY}

Let us consider $M=\left(\Omega, \mathcal{F}, \mathcal{F}_{t}, x_{t}, P_{x}\right)$ a (strong right) Markov process, the realization of an SHS. The stochastic reachability problem is defined as follows. Given a target set, the objective of the reachability problem is to compute the probability that the system trajectories from an arbitrary initial state will reach the target set. Formally, given a set $A \in$ $\mathcal{B}(X)$ and a time horizon $T>0$, let us define: $\operatorname{Reach}_{T}(A)=$ $\left\{\omega \in \Omega \mid \exists t \in[0, T]: x_{t}(\omega) \in A\right\}, \operatorname{Reach}_{\infty}(A)=\{\omega \in$ $\left.\Omega \mid \exists t \geq 0: x_{t}(\omega) \in A\right\}$.

These two sets are the sets of trajectories of $M$, which reach the set $A$ (the flow that enters $A$ ) in the interval of time $[0, T]$ or $[0, \infty)$. The reachability problem consists of determining the probabilities of such sets. The probabilities of reach events are

$$
P\left(T_{A}<T\right) \text { or } P\left(T_{A}<\zeta\right)
$$

where $\zeta$ is the life time of $M$ and $T_{A}$ is the first hitting time of $A$

$$
T_{A}=\inf \left\{t>0 \mid x_{t} \in A\right\}
$$

and $P$ is a probability on the measurable space $(\Omega, \mathcal{F})$ of the elementary events associated to $M . P$ can be chosen to be $P_{x}$ (if we want to consider the trajectories that start in $x$ ). Denote by $P_{A}$ the hitting operator associated to the underlying Markov process $\left(x_{t}\right)$, i.e. $P_{A} v=E_{x}\left\{v \circ x_{T_{A}} \mid T_{A}<\zeta\right\}$ and 
$T_{A}$ is given by (3). We have $P_{x}\left[\operatorname{Reach}_{\infty}(A)\right]=P_{A} 1(x)=$ $P_{x}\left[T_{A}<\zeta\right]$.

\section{Symmetry Reduction: Discrete Setting}

In this section, we briefly present the mathematical apparatus of symmetry reduction for discrete probabilistic models as it was developed in the literature [8].

\section{A. Deterministic case}

Let $M=(X, \mathcal{R})$ be a transition system with $X$ a finite/countable set of states and a transition relation $\mathcal{R} \subseteq$ $X \times X$. A bijective map (permutation) $\pi: X \rightarrow X$ is called an automorphism when it preserves the transition relation $\mathcal{R}$, i.e. $\left(x, x^{\prime}\right) \in \mathcal{R} \Rightarrow\left(\pi(x), \pi\left(x^{\prime}\right)\right) \in \mathcal{R}$. A group $G$ of such automorphisms generates an equivalence relation $\epsilon$ on the space $X$ (defined by $\left(x, x^{\prime}\right) \in \epsilon$ if there is permutation in $G$ mapping $x$ to $x^{\prime}$, i.e. if $x$ and $x^{\prime}$ are symmetric). $\epsilon$ is called the orbit relation, and its equivalence classes are called orbits. Let $\bar{X}$ be the set containing a unique representative state for each equivalence class, we can define a function rep $: X \rightarrow \bar{X}$ that selects the corresponding unique representative $\operatorname{rep}(x) \in \bar{X}$, for each state $x \in X$ and uses this to define a new transition relation $\overline{\mathcal{R}}=$ $\left\{\left(\operatorname{rep}(x), \operatorname{rep}\left(x^{\prime}\right)\right) \mid\left(x, x^{\prime}\right) \in \mathcal{R}\right\}$. Since all permutations in $G$ preserve the transition relation $\mathcal{R}$, the quotient transition system $(\bar{X}, \overline{\mathcal{R}})$ is bisimilar to the original transition system $(X, \mathcal{R})$.

\section{B. Probabilistic Case}

For DTMC, CTMC, the concept of symmetry can be formulated in an analogous way to the non-probabilistic case. Consider permutations of the state space $\pi: X \rightarrow X$ that preserve the transition function. For DTMC, we require that $P\left(\pi(x), \pi\left(x^{\prime}\right)\right)=P\left(x, x^{\prime}\right), \forall x, x^{\prime} \in X$. Similarly, for CTMC, we need $R\left(\pi(x), \pi\left(x^{\prime}\right)\right)=R\left(x, x^{\prime}\right), \forall x, x^{\prime} \in X$.

Consider a group $G$ of such permutations on $X$ and the corresponding orbit relation $\epsilon$. Using the equivalence w.r.t. $\epsilon$, we define a reduced state space $\bar{X}$ containing a unique representative for each orbit and a function rep $: X \rightarrow$ $\bar{X}$, which computes the representative for each state. The construction of the quotient model can be done as follows. For a DTMC $(X, P)$ we build the quotient DTMC $(\bar{X}, \bar{P})$, where for each pair of states $\bar{x}, \bar{x}^{\prime} \in \bar{X}: \bar{P}\left(\bar{x}, \bar{x}^{\prime}\right)=$ $\sum_{\left\{x^{\prime} \in X \mid r e p\left(x^{\prime}\right)=\bar{x}^{\prime}\right\}} P\left(\bar{x}, x^{\prime}\right)$. For a CTMC $(X, R)$, the quotient model is $(\bar{X}, \bar{R})$, where for $\bar{x}, \bar{x}^{\prime} \in \bar{X}: \bar{R}\left(\bar{x}, \bar{x}^{\prime}\right)=$ $\sum_{\left\{x^{\prime} \in X \mid r e p\left(x^{\prime}\right)=\bar{x}^{\prime}\right\}} R\left(\bar{x}, x^{\prime}\right)$.

In the case of DTMCs and CTMCs, the automorphisms used in symmetry reduction of the state space are invariance automophisms, i.e. they preserve the transition probabilities. Applying such automorphims to a chain, the new chain has the same law as the initial one.

\section{Symmetry REduction VIA the InVARIANCE Group: CONTINUOUS SETTING}

Note that in [8], for the Markov chains, the automorphisms defined in [8] preserve the transition system structure. For the case of continuous-time continuous space Markov processes, this system structure is no longer available (the concept of next state is available only for Markov chains). Then the concept of invariance automorphism should be different: It has to preserve the probabilistic dynamics of the system. Formally, consider a Markov process as a family $\left\{x_{t}^{x} \mid x \in\right.$ $X\}$ of processes, where $x_{t}^{x}$ is the process starting at $x$. If $\pi: X \rightarrow X$ is a homeomorphism, then $\pi\left(x_{t}\right)$ is also a Markov process. The transformation $\pi$ is called invariance automorphism of $x_{t}$ if the process $\pi\left(x_{t}^{x}\right)$ is identical in law with $x_{t}^{\pi(x)}$.

\section{A. Invariance}

Consider a continuous Markov process defined as in Subsection II-B. Suppose that $\pi$ is a measurable one to one transformation of the state space $(X, \mathcal{B})$. Then we can identify the Wiener probabilities $\widetilde{P}_{x}=P_{\pi^{-1}(x)}$ on $\mathcal{F}$. The transformed process is of the form $\widetilde{M}=\left(\pi x_{t}, \zeta, \mathcal{F}_{t}, P_{\pi^{-1}(x)}\right)$. Its transition function is defined by the formula $\widetilde{p}_{t}(x, \Gamma)=$ $p_{t}\left(\pi^{-1}(x), \pi^{-1} \Gamma\right)$. We say that a Markov process $M$ is invariant w.r.t. a transformation $\pi$, if the following conditions are satisfied: (i) For each $\omega \in \Omega$, there exists $\omega^{\prime} \in \Omega$ such that $\pi x_{t}(\omega)=x_{t}\left(\omega^{\prime}\right)$ for all $0 \leq t<\zeta(\omega)=\zeta\left(\omega^{\prime}\right)$. (ii) For all $t>0, x \in X, \Gamma \in \mathcal{B} p_{t}(x, \Gamma)=p_{t}\left(\pi^{-1}(x), \pi^{-1} \Gamma\right)$. If a Markov process $M$ is invariant w.r.t. $\pi$, then the transformed process $\widetilde{M}$ is equivalent to $M$ [6]. If $B$ is a set of trajectories, we can define the shift $\theta_{\pi} B$ (w.r.t. $\pi$ ) as follows. Put $\omega \in \theta_{\pi} B$, if $\omega^{\prime}$ can be found such that (i) holds. Then $\theta_{\pi}\left\{x_{t} \in \Gamma\right\}=\left\{\pi x_{t} \in \Gamma\right\}=\left\{x_{t} \in \pi^{-1} \Gamma\right\}$, for any $t \geq 0$, $\Gamma \subseteq X$.

Theorem 1 (Invariance of the Wiener Probabilities):

[6] Let $M$ be a Markov process on the state space $(X, \mathcal{B})$ invariant $\quad$ w.r.t. a transformation $\pi$. Then $P_{\pi^{-1} x}\left(\theta_{\pi} A\right)=P_{x}(A)$, for each $A \in \mathcal{F}$ and $x \in X$.

The transformation $\pi$ that appears in the definition of invariance can be called invariance automorphism of $M$. This preserves the transition probabilities and transforms a trajectory of $M$ into another one.

\section{B. Symmetry reduction}

Let $M$ be a Markov process on the state space $(X, \mathcal{B})$ and let $\mathcal{G}(X)$ be a group of invariance automorphims of $M$. Suppose that the group $\mathcal{G}$ preserves the measurable sets. This group generates an equivalence relation $\epsilon$ on the space $X$, defined by $\left(x, x^{\prime}\right) \in \epsilon$ if there exists an automorphism in $\mathcal{G}$ mapping $x$ to $x^{\prime}$. The subsets $\{\mathcal{G} x\}_{x \in X}$ are called orbits of the group $\mathcal{G}$. Denote by $\widetilde{X}:=X / \mathcal{G}$ the set of all orbits of the group $\mathcal{G}$. Denote by $\gamma$ the projection map from $X$ to $\widetilde{X}$ defined by $\gamma x:=\{\mathcal{G} x\}$. Let $\widetilde{\mathcal{B}}:=\mathcal{\gamma} \mathcal{B}$. then $\gamma$ is a measurable transformation of $(X, \mathcal{B})$ into $(\widetilde{X}, \widetilde{\mathcal{B}})$. The invariance of $M$ w.r.t. to the automorphims of $\mathcal{G}$ enables us to construct a Markov process on the state space $(\widetilde{X}, \widetilde{\mathcal{B}})$ from the Markov process $M$, using the transformation $\gamma$ [6]. Denote by $M / \mathcal{G}$ this new Markov process. $M / \mathcal{G}$ is obtained from $M$ by symmetry reduction of the state space w.r.t. the group $\mathcal{G}$.

We can define a reduced state space or a fundamental domain for the group $\mathcal{G}$ as follows. A set $\bar{X} \subset X$ is a 
reduced state space for $\mathcal{G}$ if one and only one point belonging to $\bar{X}$ can be found in each orbit $\{\mathcal{G} x\}$. Then associating the class $\{\mathcal{G} x\}$ with this point we obtain a one to one mapping $\beta: \widetilde{X} \rightarrow \bar{X}$. Naturally, we can then define $\operatorname{rep}: X \rightarrow \bar{X}$; rep $:=\beta \circ \gamma$. Assume that $\bar{X} \in \mathcal{B}$ and set $\overline{\mathcal{B}}:=\mathcal{B}(\bar{X})$. Then $\beta \widetilde{\mathcal{B}}=\overline{\mathcal{B}}$ and $\beta^{-1} \overline{\mathcal{B}}=\widetilde{\mathcal{B}}$. This says that it is possible to identify the space $(\widetilde{X}, \widetilde{\mathcal{B}})$ with the space $(\bar{X}, \overline{\mathcal{B}})$ and consider the process $M / \mathcal{G}$ to be given on $(\bar{X}, \overline{\mathcal{B}})$. The Markov process $M$ under $P_{x}$ is equivalent with the Markov process $M / \mathcal{G}$ under $\bar{P}_{r e p(x)}$.

\section{SyMmetry Reduction Via Symmetry Groups: CONTINUOUS SETTING}

When we are considering complex Markov processes as those that appear as semantics of SHS, the symmetry reduction described in the Section $\mathrm{V}$ might be difficult to apply. We need to find an appropriate transformation group $\mathcal{G}$ whose elements are also automorphisms for the diffusion components. As well, we need to check properties like the invariance of the transition rate $\lambda$ or of the stochastic kernel $R$ (that appear in the definition of SHS) w.r.t. the elements of $\mathcal{G}$. This might be a difficult task considering the structure of the SHS executions. In order to have two "symmetric" trajectories, we need some symmetry also for their diffusion parts. But if we start in a mode with two symmetric diffusion paths, after the first jump we may get some asymmetric paths in another mode or in two different modes.

Our novelty is to replace invariance groups by transformation groups for which we have the symmetry properties of some observation functions. These are symmetry groups and their elements are called symmetry automorphims. Formally, consider a Markov process $\left\{x_{t}^{x} \mid x \in X\right\}$. A homeomorphism $\pi: X \rightarrow X$ is called symmetry automorphism of $x_{t}$ if the process $\pi\left(x_{t}^{x}\right)$ is identical in law with $x_{t}^{\pi(x)}$ after a time change.

The line of this section can be described as follows. We present first the concept of time change for Markov processes. Then we define formally the observation functions as expectations of some random variables over the paths (that provide "observations" about the trajectories). The next step is to define the observation automorphisms as permutations of the state space that preserve the observation functions. The group of such automorphisms is used thereafter to "reduce the state space" considering the quotient space w.r.t. the equivalence relation induced by this group. At the end, we show that this symmetry reduction of state space preserves the reach set probabilities.

\section{A. Time change}

Let us recall briefly the definition of time changes for Markov processes [11]. A real valued process $A_{t}$ is called an additive functional of $\left(x_{t}\right)$ if it is adapted to the natural filtration of $\left(x_{t}\right)$ and satisfies $A_{0}=0$ and $A_{t+s}=A_{t}+A_{s} \circ \theta_{t}$, where $\theta_{t}$ is the shift operator defined by (1). Suppose that an additive functional has continuous strictly continuous paths. Let $\tau_{t}$ be the inverse of $A_{t}$ considered as a function of $t . \tau_{t}$ is called a time-change process of $\left(x_{t}\right)$. The process $\left(x_{\tau_{t}}\right)$ (which is also a Markov process) has the same physical paths as $\left(x_{t}\right)$, but runs according to a different clock.

Let $a(x)$ a positive continuous function on $X$ bounded away from 0 . Then $A_{t}=\int_{0}^{t} a\left(x_{s}\right) d s$ is an additive functional and $a$ is called the density of $A_{t}$. If $\tau_{t}$ is the inverse of $A_{t}$, then the time-changed process $\left(x_{\tau_{t}}\right)$ is said to be obtained from $\left(x_{t}\right)$ by the time change with density $a$. In this case, the generator of the time-changed process is given by (see [11], p.278): $\widetilde{\mathcal{L}} f(x)=a(x)^{-1} \mathcal{L} f(x) ; f \in D(\mathcal{L})$.

Two processes that differ by a time change have the same hitting distribution, by the Blumenthal-Getoor-McKean Theorem (Ch. 5 of [3]). Then, two such processes have the same reach set probabilities, so they are "bisimilar".

\section{B. Observability over the paths}

We suppose that the trajectories $x:[0, \infty) \rightarrow X$ of $M$ are cadlags. We consider $\Omega=D_{X}[0, \infty)$, the set of all these paths (i.e. the space of all cadlag functions from $[0, \infty)$ to $X)$. In the following, we define a special class of functions called observation functions for the Markov process $M$. These functions play the role of some logic formulas over the trajectories. First we define the observation random variables. Taking the expectations of such random variables represents a technique to generate observation functions. This technique provides also intuitions about the meaning of these functions.

A nonnegative function $\eta: \Omega \rightarrow \mathbb{R}_{+}$is said to be an observation random variable for the process $M$, if: (i) the function $\eta$ is measurable; (ii) the value of $\eta$ on the shifted trajectory is less than the value of $\eta$ on the whole trajectory, i.e. $\eta\left(\theta_{t} \omega\right) \leq \eta(\omega)$ for all $0 \leq t<\zeta(\omega)$; (iii) the function $\eta\left(\theta_{t} \omega\right)$ is right-continuous in $t \in[0, \zeta(\omega))$ for all $\omega$. In the language of [6], the observation random variables are called excessive random variables. Let $\eta$ be an excessive random variable, satisfying the additional requirement: $0<E_{x} \eta<$ $\infty$, for all $x \in X$.

Proposition 2: Let $M$ be a strong Markov process. If $\eta$ is an observation random variable, then $f(x)=E_{x} \eta$ satisfies the following conditions: (a) $E_{x} f\left(x_{\tau}\right) \leq f(x)$, for all $x \in X$ and for any stopping time $\tau$; (b) $\lim _{n \rightarrow \infty} E_{x} f\left(x_{\tau_{n}}\right)=f(x)$, for any $x \in X$ and any sequence of stopping times $\tau_{n}$ such that $P_{x}\left(\tau_{n} \searrow 0\right)=1$.

If $h$ is an arbitrary non-negative $\mathcal{B}$-measurable function then $\eta=\int_{0}^{\zeta} h\left(x_{t}\right) d t$, where $\zeta$ is the life time of $M$, is an observation random variable.

The set of non-negative measurable functions $f$ that satisfy the conditions (a) and (b) from the Prop. 2 may be larger than the set of such functions provided by observation random variables. For instance, these properties remain true for limits of such function

A non-negative measurable function $f: X \rightarrow[0, \infty]$ is called observation function for the process $M$ if the conditions (a) and (b) from the Prop. 2 are fulfilled.

Theorem 3: A non-negative measurable function $f: X \rightarrow$ $[0, \infty]$ is an observation function for a strong Markov process $M$ iff the following conditions w.r.t. the operator semigroup $\mathcal{P}$ are satisfied: (i) $P_{t} f(x) \leq f(x)$ for all $t \geq 0, x \in X$; (ii) $P_{t} f(x) \rightarrow f(x)$ as $t \searrow 0$, for every $x \in X$. 
Th.3 shows that our observation functions are exactly 0 -excessive functions defined in the context of Markov processes. Let us denote by $\mathcal{O} b(M)$ the set of observation (or 0 -excessive) functions associated to $M$. Recall that a function $f$ is called $\alpha$-excessive ( $\alpha \geq 0$ ) w.r.t. the semigroup $\left(P_{t}\right)$ if it is measurable, non-negative and $e^{-\alpha t} P_{t} f \leq f$, for all $t \geq 0$ and $e^{-\alpha t} P_{t} f \nearrow f$ as $t \searrow 0$. Let $\mathcal{E}_{M}^{\alpha}$ be the set of all excessive functions associated to $M$. According to the Blumenthal-Getoor-McKean theorem [3], the cone of excessive functions determines the process up to a time change. We assume also that $M$ is transient [3]. This hypothesis guarantees that the cone $\mathcal{O} b(M)$ is rich enough to be used.

\section{Symmetry Group}

Let us consider a transient Markov process $M$ with the state space $(X, \mathcal{B})(M$ as the realization of an SHS, $H)$. Let $\mathcal{S}(X)$ be the group of all homeomorphisms $\varphi: X \rightarrow X$, i.e. all bijective maps $\varphi$ such that $\varphi, \varphi^{-1}$ are $\mathcal{B}(X)$-measurable. When $X$ is finite, $\mathcal{S}(X)$ is the set of (finite) symmetries of $X$. Any symmetry ${ }^{2}$ of $X$ induces a symmetry of $\mathbf{B}(X)$ as follows. Let $*: \mathcal{S}(X) \rightarrow \operatorname{Perm}[\mathbf{B}(X)]$ be the action $\mathcal{S}(X)$ to $\mathbf{B}(X)$ defined by $*(\varphi)=\varphi^{*}: \mathbf{B}(X) \rightarrow \mathbf{B}(X)$, where $\varphi^{*}$ is the linear operator on $\mathbf{B}(X)$ given by $\varphi^{*} f=f \circ \varphi$. The range of ' $*$ ' is enclosed in $\operatorname{Perm}[\mathbf{B}(X)]$ (the symmetry group of $\mathbf{B}(X)$ ). Then, $\varphi^{*}$ can be thought of as a symmetry of $\mathbf{B}(X)$ for each $\varphi$.

Clearly $\mathcal{O} b(M) \subset \mathbf{B}(X)$. We can not define the action ' $*$ ' of $\mathcal{S}(X)$ to $\mathcal{O} b(M)$ because $*(\mathcal{O} b(M)) \varsubsetneqq \mathcal{O} b(M)$. It is then necessary to consider those subgroups of $\mathcal{S}(X)$ such that we can define the action of these subgroups on the semigroup $\mathcal{O} b(M)$. Consider the maximal subgroup of symmetries of the state space $X$, denoted by $\mathcal{H}$, such that the action of $\mathcal{H}$ to $\mathcal{O} b(M)$ denoted also by ' $*$ ' can be defined: * : $\mathcal{H} \rightarrow \operatorname{Perm}[\mathcal{O} b(M)]$. We have the invariance of the observations w.r.t. the elements of $\mathcal{H}$. These observations could be interpreted as well as some stochastic specifications of the system. $\mathcal{H}$ is not necessary to be taken as the maximal subgroup of symmetries with this property. Naturally, the elements of $\mathcal{H}$ will be called observation automorphisms of $M$.

Using $\mathcal{H}$, an equivalence relation $\mathcal{O} \subset X \times X$, called observation relation, can be defined on the state space $X$ as follows: Two states $x, y$ are in the same orbit, written $x \mathcal{O} y$, if and only if there exists an observation automorphism $\varphi \in \mathcal{H}$ such that $\varphi(x)=y$. Let us denote by $[x]$ the equivalence class containing the point $x$ in $X$. The equivalent classes of $\mathcal{O}$ are called orbits. It is clear that an orbit $[x]$ can be described as $[x]=\{\varphi(x) \mid \varphi \in \mathcal{H}\}=\{\mathcal{H} x\}$. Let $X / \mathcal{O}$ denote the set of orbits, and let $\Pi_{\mathcal{O}}$ the canonical projection $\Pi_{\mathcal{O}}: X \rightarrow X / \mathcal{O}, \Pi_{\mathcal{O}}(x)=[x]$. The space $X / \mathcal{O}$ will be equipped with the quotient topology by declaring a set $A \subset X / \mathcal{O}$ to be open if and only if $\Pi_{\mathcal{O}}^{-1}(A)$ is open in $X$. $\Pi_{\mathcal{O}}$ is a continuous map w.r.t. the initial topology of $X$ and the quotient topology of $X / \mathcal{O}$.

\footnotetext{
${ }^{2}$ Here, permutation is used with the sense of one-to-one correspondence or bijection.
}

\section{Symmetry Reduction}

In this subsection, we show that the observation automorphisms are, in fact, symmetry automorphims, so they preserve the hitting distributions. Consequently, the reach set probabilities (2) are preserved through the observation automorphisms. Moreover, since the reach set probabilities are preserved, the observation relation $\mathcal{O}$ is nothing else, but a bisimulation relation on the state space.

Proposition 4: Let $g: X / \mathcal{O} \rightarrow \mathbb{R}$ be a $\mathcal{B}(X / \mathcal{O})$ measurable and let $E=\Pi_{\mathcal{O}}^{-1}(A)$ for some $A \in \mathcal{B}(X / \mathcal{O})$. Then the following equality holds

$$
P_{E}=\varphi^{*} \circ P_{A}, \forall \varphi \in \mathcal{H}
$$

applied to all functions $f: X \rightarrow \mathbb{R}, f=g \circ \Pi_{\mathcal{O}}$.

Corollary 5: Any observation automorphism $\varphi \in \mathcal{H}$ for $M$ is a symmetry automorphism, i.e. $M$ and $\varphi(M)$ differ by a time change, then they have the same hitting distributions.

Formula (4) shows that the function $P_{E} f$ (where $f=$ $\left.g \circ \Pi_{\mathcal{O}}\right)$ is constant on the orbits of $\mathcal{O}$. Then it makes sense to define a collection of operators $\left(Q_{A}\right)$ on $(X / \mathcal{O}, \mathcal{B}(X / \mathcal{O}))$ by setting $Q_{A} g([x])=P_{E}\left(g \circ \Pi_{\mathcal{O}}\right)(x)$, where $E=\Pi_{\mathcal{O}}^{-1}(A)$ (Prop.4 allows to use any representative $x$ of $[x]$ in the right side of this equality). It is easy to check that $Q_{A} Q_{B}=Q_{B}$ if $A$ and $B$ are open sets of $X / \mathcal{O}$ with $B \subset A$. Under some supplementary hypotheses one can construct a Markov process $M / \mathcal{O}=\left([x]_{t}, Q_{[x]}\right)$ with these hitting operators [3]. $M / \mathcal{O}$ is obtained from $M$ by symmetry reduction of the state space w.r.t. the group $\mathcal{H}$ and the set of observations $\mathcal{O} b(M)$.

\section{E. Stochastic Bisimulation}

For a continuous time continuous space Markov process $M$ with the state space $X$, an equivalence relation $\mathcal{R}$ on $X$ is called (strong) bisimulation if for $x \mathcal{R} y$ we have $p_{t}(x, A)=$ $p_{t}(y, A), \forall t>0, \forall A \in \mathcal{B}\left(X /{ }_{R}\right)$, where $p_{t}(x, A), x \in X$ are the transition probabilities of $M$ and $\mathcal{B}\left(X /{ }_{R}\right)$ represent the $\sigma$-algebra of measurable sets closed w.r.t. $\mathcal{R}$. This variant of strong bisimulation considers two states to be equivalent if their 'cumulative' probability to 'jump' to any set of equivalent classes (that this relation induces) is the same. This is hard to be checked in practice since the time $t$ runs continuously. Then a robust bisimulation relation on $X$ must be defined as a relation that preserves only the measures of interest for the Markov process $M$.

In the following we briefly present a more robust concept of bisimulation defined in [5]. Suppose we have given a Markov process $M$ on the state space $X$, w.r.t. a probability space $(\Omega, \mathcal{F}, \mathbf{P})$. Assume that $\mathcal{R} \subset X \times X$ is an equivalence relation such that the quotient process $\left.M\right|_{\mathcal{R}}$ is still a Markov process with the state space $X / \mathcal{R}$, w.r.t. a probability space $(\Omega, \mathcal{F}, \mathbf{Q})$. A relation $\mathcal{R}$ is called behavioral bisimulation on $X$ if for any $A \in \mathcal{B}(X / \mathcal{R})$ we have that $\mathbf{P}\left[T_{E}<\right.$ $\infty]=\mathbf{Q}\left[T_{A}<\infty\right]$, where $E=\Pi_{\mathcal{R}}^{-1}(A)$ (i.e. the reach set probabilities of the process $M$ and $\left.M\right|_{\mathcal{R}}$ are equal).

Theorem 6: The observation relation $\mathcal{O}$ is a bisimulation relation on $(X, \mathcal{B})$ for the Markov process $M$. 
Th. 6 is a simple consequence of the Prop. 4, but its statement is very important in the context of stochastic reachability. It states that the symmetry reduction of the state space defined via observation automorphisms represents a sound approach that can be used further in stochastic model checking.

\section{TOWARDS SYMMETRY REDUCTION FOR SHS}

In this section, we discuss how the symmetry reduction techniques described in Sections V and VI can be further adapted in the framework of stochastic hybrid systems. We have already pointed out that the fact that symmetry reduction via invariance groups is not a realistic choice for SHS due to the jumping mechanism between the discrete locations. One way to deal with this method is to apply symmetry reduction locally in each mode for the corresponding diffusion process and then to find the appropriate composition mechanism for these local abstractions, in order to obtain the global abstraction of the given SHS.

The second symmetry reduction technique (via a group of observation automorphisms, Section VI) might be a valuable method to reduce the state space of a stochastic hybrid system. The efficiency of this method depends pretty much on our ability to choose the generators of the semigroup of observation functions. Considering the connection between the semigroup of operators and the infinitesimal generator of a Markov process based on the Th.3, one can easily obtain characterizations of the observation functions in terms of the generator.

The infinitesimal generator of the realization of an SHS $H$ is an integro-differential operator. The extended generator of an SHS has the following expression:

$$
\mathcal{L} f(x)=\mathcal{L}_{\text {cont }} f(x)+\mathcal{L}_{\text {dis }} f(x)
$$

where $\mathcal{L}_{\text {cont }} f(x)$ has the standard form of the diffusion infinitesimal operator and $\mathcal{L}_{\text {dis }} f(x)=\lambda(x) \int_{\bar{X}}(f(y)-$ $f(x)) R(x, d y)$ (typical generator of a jump process). The domain $D(\mathcal{L})$ contains at least the set of second order differentiable functions that satisfy the following boundary condition: $f(x)=\int_{\mathbb{X}} f(y) R(x, d y), \quad x \in \partial X$. For any $\varphi \in \mathcal{S}(X)$ (where $\mathcal{S}(X)$ is defined as in Subsection VI$\mathrm{C})$, the generator of $\varphi(M)$ is given by $\mathcal{L}^{\varphi} f=\varphi_{*}\left[\mathcal{L}\left(\varphi^{*} f\right)\right]$, where $\varphi_{*} f:=f \circ \varphi^{-1}$. Then we can define the invariance group $\operatorname{Inv}(\mathcal{L}):=\left\{\varphi \in \mathcal{S}(X) \mid \mathcal{L}^{\varphi}=\mathcal{L}\right\}$. Analogously, the symmetry group can be defined taking into account the results from Subsection VI-A as follows: $\operatorname{Sym}(\mathcal{L}):=$ $\left\{\varphi \in \mathcal{S}(X) \mid \exists \beta \in C_{0}(X), \beta>0, \mathcal{L}^{\varphi}=\beta \mathcal{L}\right\}$. Clearly, $\operatorname{Inv}(\mathcal{L}) \subset \operatorname{Sym}(\mathcal{L})$. To apply symmetry reduction to SHS, we need the assumption that there is a group of symmetries acting uniformly on the diffusion processes of different discrete modes, and the transition rate $\lambda$ and the stochastic kernel $R$ are 'invariant' w.r.t. these symmetries. Finding appropriate symmetry automorphisms for SHS might be a difficult and challenging task. In the first step, considering the expression of the SHS generator (5), it is clear that we need to consider symmetry groups for the continuous dynamics of an SHS. Characterizations of the invariance group and symmetry group for diffusion processes can be given using the isometry group (that consists of transformations which leave the metric invariant) and the conformal group (that consists of transformations which do not change the angles) [9]. In the second step, consider $\varphi$ a symmetry/invariant automorphism for the diffusion part and observe that

$$
\begin{gathered}
\mathcal{L}_{\text {dis }}^{\varphi} f(x) \\
f(x)\} .
\end{gathered}
$$

Proposition 7: $\varphi$ is an invariant automorphism for the whole process $M_{H}$ (realization of $H$ ) iff $\varphi_{*} \lambda=$ $\lambda, \int_{\bar{X}} f(\varphi(y)) R\left(\varphi^{-1}(x)=\int_{\bar{X}} f(y) R(x, d y), f \in D(\mathcal{L})\right.$ A similar condition can be written for a symmetry automorphism. In a upcoming paper, we will investigate further these conditions in order to find necessary conditions for a transformation group to be an appropriate subgroup of $\operatorname{Inv}(\mathcal{L})$ or $\operatorname{Sym}(\mathcal{L})$, where $\mathcal{L}$ is the infinitesimal generator of an SHS.

\section{CONCLUSIONS}

Modelling with SHS is very fashionable in engineering because of the versatile randomisation techniques it offers. However, this paradigm is less popular in computer science due to the inherent complexity of the formal verification of safety properties. In this work, we address the verification issue by investigating how probabilistic model checking techniques from computer science can be extending for SHS. We have mainly presented two techniques for symmetry reduction of the state space for continuous probabilistic systems. Both of them are based on the same methodology to obtain the reduced state space: choose an appropriate group of permutations of the state space (the invariant group and the symmetry group) and then construct the quotient space w.r.t. this group. We have also proved that the reduced quotient model is bisimulation-equivalent to the original model. Finally, both techniques are discussed for stochastic hybrid systems.

\section{REFERENCES}

[1] Ames, A.D., Sastry, S.: Hybrid Geometric Reduction of Hybrid Systems. In Proc. IEEE CDC (2006).

[2] Blom, H.A.P., Lygeros, J. (Eds.): "Stochastic Hybrid Systems: Theory and Safety Critical Applications". LNCIS 337 (2006).

[3] Blumenthal, R.M., Getoor, R.K.: "Markov Processes and Potential Theory", Academic Press, New York and London (1968).

[4] Bujorianu, M.L., Lygeros, J.: Towards Modelling of General Stochastic Hybrid Systems. In [2]: 3-30.

[5] Bujorianu, M.L., Lygeros, J., Bujorianu, M.C.: Abstractions of Stochastic Hybrid System. Proc. 44th Conference in Decision and Control. IEEE Press (2005).

[6] Dynkin, E.B.: “Markov Processes. Vol. I\&II”. Springer Verlag (1965).

[7] Hu, J., Sastry, S.: Symmetry Reduction of a Class of Hybrid Systems. LNCS 2289. HSCC (2002): 267 - 280

[8] Kwiatkowska, M., Norman, G., Parker, D.: Symmetry Reduction for Probabilistic Model Checking. In Proc. 18th International Conference on Computer Aided Verification (CAV'06), LNCS 4144, (2006): 234248.

[9] Liao, M.: Symmetry Groups of Markov Processes. Ann. Prob. 20 (2), (1992): 563-578.

[10] Miller, A, Donaldson, A.F., Calder, M.: Symmetry in Temporal Logic Model Checking. ACM Computing Surveys (CSUR). 38 (3) (2006).

[11] Williams, D., Rogers, L.C.G.: "Diffusions, Markov Processes, and Martingales: Volume 1, Foundations". Cambridge Mathematical Library (2000). 